\title{
Analysis of Multiple-Beam Fizeau Fringes Crossing Quasi-D-shaped Optical Fiber
}

\author{
M. M. El-Nicklawy, R. M. El-Agmy ${ }^{*}$, A. F. Hassan, \\ M. El-Hagary and Amany Adel \\ Helwan University, Faculty of Science, Physics department, \\ Helwan, Egypt
}

Mathematical expression for multiple-beam Fizeau fringes crossing quasi-D-shaped optical fiber is derived. The numerical simulations for the derived equations at different fiber rotations angles have been presented. Experimental validation for the derived equations is verified. Microinterferograms for Fizeau fringes crossing the fiber in transmission is also presented.

\section{Introduction:}

High power fiber lasers are needed for many industrial applications. Dshaped fiber lasers have been used as a high fiber lasers source due to better absorption inside the fiber core even when using multimode pump source [1-2]. Refractive indices of the fiber core-cladding and core diameter are the basic transmission parameter. Adjustment of these parameters is major of interest during and after manufacturing process. Different techniques have been used to determine refractive indices and core-cladding dimensions [3-7]. For fibers having perfectly regular transverse cross-section, theories have been presented [8-12], for both single and multiple-index fibers. The interferometric methods applied to fibrous material has been discussed extensively [12-15]. Mathematical expressions for multiple-beam Fizeau fringes for regular and irregular crosssections have been presented by El-Nicklawy et al. [10, 12, 14 \& 15].

In the present work, the analysis has been extended to derive the mathematical formulas for Fizeau fringes for fibers that having elliptical core and quasi-D-shaped cladding. Numerical modeling for the derived equations will be introduced and experimental validation of the derived equation will be given.

\footnotetext{
* Corresponding author, e-mail: Reda-Elagamy@Helwan.edu.eg
} 


\section{Theoretical Considerations:}

The theoretical model is driven based on an electron micrograph of the fiber as shown in Fig. (1). Consider a fiber of elliptical core of radii a and b, two crossed circles of radii R1 and R1 to form quasi-D-Shaped cladding as shown in Fig. (2). Let the fiber core of refractive index $n_{c}$ and its cladding of refractive index $n_{s}$ be introduced in a silvered liquid wedge of refractive index $n_{L}$ with wedge angle $\varepsilon$.

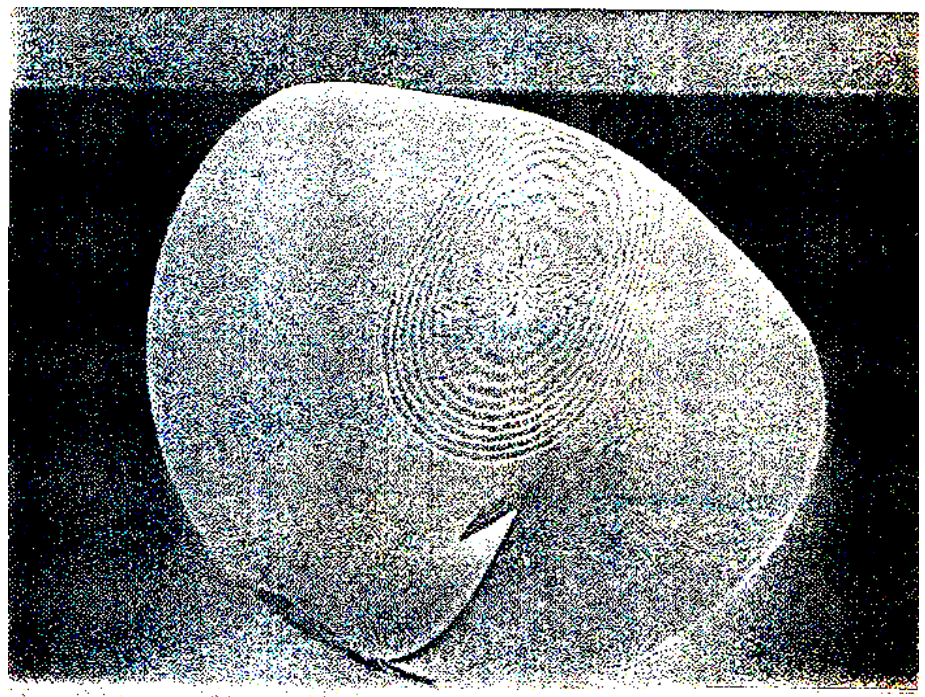

Fig. (1): Scanning electron microscope picture of the fiber.

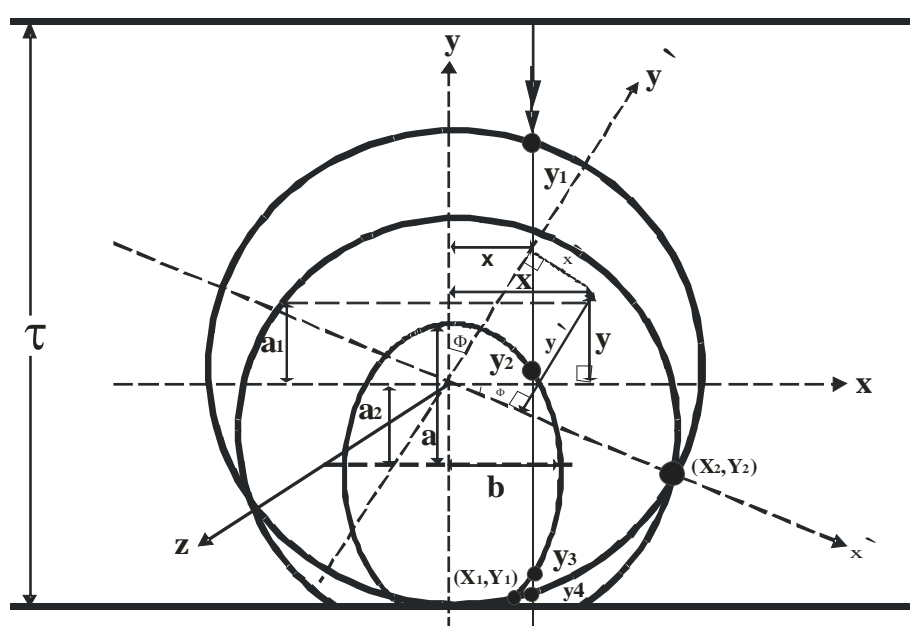

Fig. (2): Cross-section of a fiber having an elliptical core, with a refractive index $n_{c}$, shifted away from the centre of a skin of quasi-D-shaped of radii $R_{1}$ and $R_{2}$, of refractive index $n_{s}$, immersed in a silvered liquid wedge of refractive index $n_{L}$. 
Let the setting of the fiber be such that its axis (the z-axis) is perpendicular to the edge of the wedge (the $\mathrm{x}$-axis). A monochromatic light beam of wavelength $\lambda$ traverses the fiber parallel to the major radius of its core. The optical path length, OPL, through the $x-y$ plane, (Fig. (2)) will be given by:

$$
O P L=n_{L} t+\left(y_{1}-y_{2}\right)\left(n_{s}-n_{L}\right)+\left(y_{2}-y_{3}\right)\left(n_{c}-n_{L}\right)+\left(y_{3}-y_{4}\right)\left(n_{s}-n_{L}\right)
$$

where $\mathrm{t}=\mathrm{z} \tan \varepsilon$.

$$
\begin{aligned}
& y_{1}=\left(R_{1}^{2}-x^{2}\right)^{1 / 2} \\
& y_{2}=a \sqrt{1-\frac{x^{2}}{b^{2}}}-a_{2} \\
& y_{3}=-a \sqrt{1-\frac{x^{2}}{b^{2}}}-a_{2} \\
& y_{4}=-\sqrt{R_{2}^{2}-x^{2}}+a_{1}
\end{aligned}
$$

where $\mathrm{R}_{1}$ and $\mathrm{R}_{2}$ are the radii of the fiber and $\mathrm{a}$ and $\mathrm{b}$ are the major and minor radii of the elliptical core, respectively.

For a fringe of interference of order $N$ we get:

$$
N \lambda=2\left[n_{L} t_{N}+\left(y_{1}-y_{2}\right)\left(n_{s}-n_{L}\right)+\left(y_{2}-y_{3}\right)\left(n_{c}-n_{L}\right)+\left(y_{3}-y_{4}\right)\left(n_{s}-n_{L}\right)\right]
$$

Transforming the origin to $\left(0, \frac{N \lambda}{2 n_{L} \tan \varepsilon}\right)$ on the $x-z$ plane gives:

$n_{L} z \tan \varepsilon=\left(y_{1}-y_{2}\right)\left(n_{s}-n_{L}\right)+\left(y_{2}-y_{3}\right)\left(n_{c}-n_{L}\right)+\left(y_{3}-y_{4}\right)\left(n_{s}-n_{L}\right)$

The ratio between the fringe shift $d z$, and the fringe spacing $\Delta z$ expressed by:

$\Delta z=\frac{\lambda}{2 n_{L} \tan \phi}$

is given by:

$$
\frac{d z}{\Delta z}=\frac{2}{\lambda}\left[\left(y_{1}-y_{2}\right)\left(n_{s}-n_{L}\right)+\left(y_{2}-y_{3}\right)\left(n_{c}-n_{L}\right)+\left(y_{3}-y_{4}\right)\left(n_{s}-n_{L}\right)\right]
$$


Equation (9) is applicable only for the following conditions, where:

$$
\begin{aligned}
& y_{1} \stackrel{\text { in-the-range }}{\longrightarrow}-R_{1} \leq x \leq R_{1} ; \\
& y_{2} \stackrel{\text { in-the-range }}{\longrightarrow}-b \leq x \leq b ; \\
& y_{3} \stackrel{\text { in-the-range }}{\longrightarrow}-\sqrt{R_{2}^{2}-\left(Y_{1}-a_{1}\right)^{2}} \geq x \geq \sqrt{R_{2}^{2}-\left(Y_{1}-a_{1}\right)^{2}} \text { and } \\
& y_{4} \stackrel{\text { in-the-range }}{\longrightarrow}-X_{2} \leq x \leq X_{2} \\
& \mathrm{X}_{2} \text { and } Y_{1} \text { are given by: } \\
& Y_{1}=\frac{-B \pm \sqrt{B^{2}-4 A C}}{2 A}
\end{aligned}
$$

where:

$$
\begin{aligned}
& \mathrm{A}=\frac{1}{\mathrm{a}^{2}}+\frac{1}{\mathrm{~b}^{2}}, \mathrm{~B}=2\left(\frac{\mathrm{a}_{1}}{\mathrm{~b}^{2}}+\frac{\mathrm{a}_{2}}{\mathrm{a}^{2}}\right) \quad \text { and } C=\frac{R_{2}^{2}}{b^{2}}-\frac{a_{1}^{2}}{b^{2}}+\frac{a_{2}^{2}}{b^{2}}-1 \\
& X_{2}= \pm \sqrt{R_{1}^{2}-\left\{\frac{a_{1}^{2}\left(R_{2}^{2}-R_{1}^{2}\right)}{2 a_{1}}\right\}^{2}}
\end{aligned}
$$

By rotating the fiber by an angle $\Phi$ the new coordinates are given by:

$$
\begin{gathered}
\left\{\begin{array}{l}
\mathrm{x}=\mathrm{y}^{\prime} \sin \phi+\mathrm{x}^{\prime} \cos \phi \\
\mathrm{y}=\mathrm{y}^{\prime} \cos \phi-\mathrm{x}^{\prime} \sin \phi
\end{array}\right\} \\
\text { or } \\
\left\{\begin{array}{l}
\mathrm{x}^{\prime}=\mathrm{x} \cos \phi-\mathrm{y} \sin \phi \\
\mathrm{y}^{\prime}=\mathrm{x} \sin \phi+\mathrm{y} \cos \phi
\end{array}\right\}
\end{gathered}
$$

Figure (3 a \& b) shows the shape of the calculated fringe shift according to Eqn. (9) at two different rotation angles. 


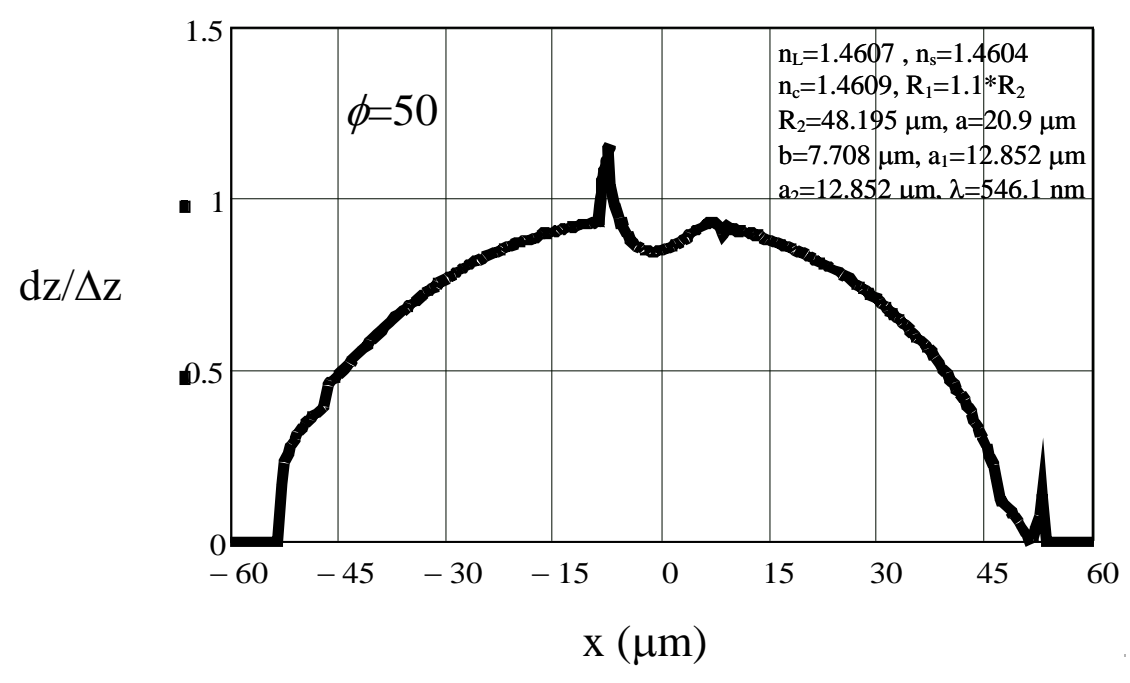

(a)

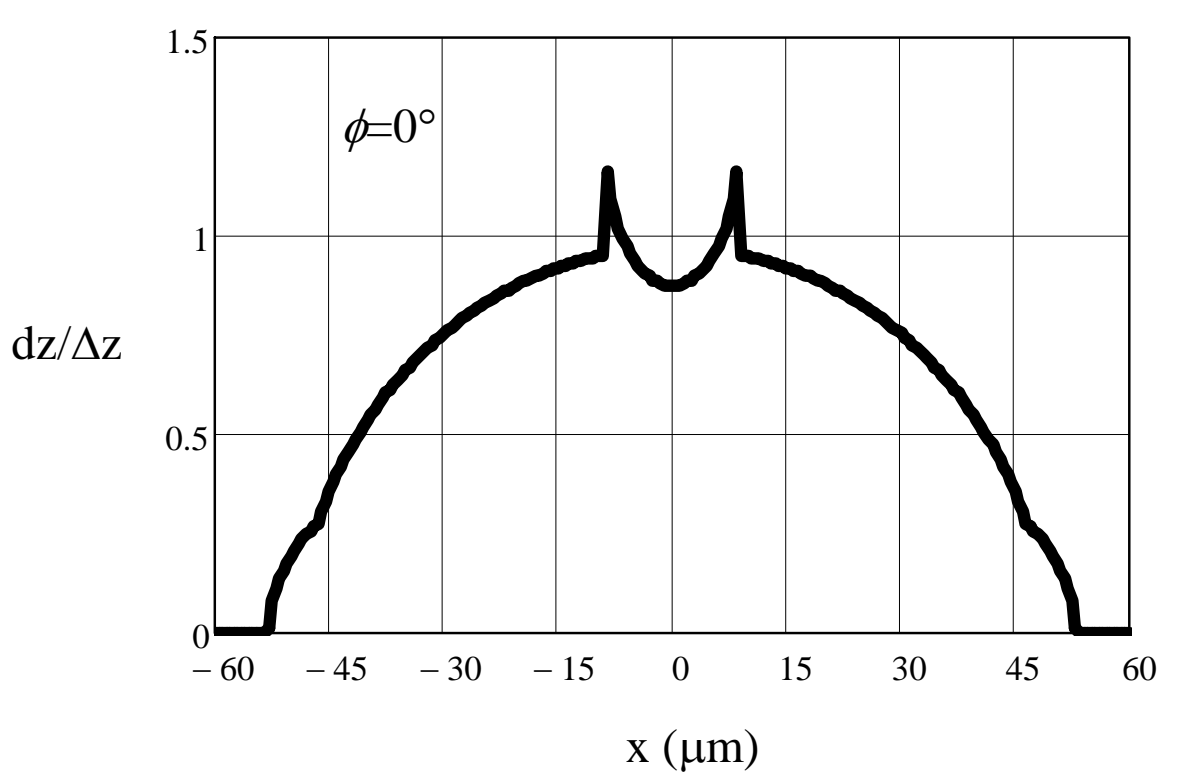

Fig. (3): Schematic diagram of the calculated $\left\lfloor\frac{d z}{\Delta z}\right\rfloor$ according to Eqn. (9). 


\section{Experimental Results:}

The interferometric was utilized to validate the numerical modeling of the derived equations. The multiple-beam Fizeau technique was described in detail [8-14]. Fig. (4) shows the microinterferograms of multiple-beam Fizeau fringes crossing the quasi-d-shaped fiber. Table (1) gives the experimental values of the refractive indices of the cladding and core for the fiber for light vibrating parallel to the fiber axis. The measured microinterferograms is in a good agreement with the calculated results shown with rotation angle of $0^{\circ}$.

Table (1): Values of refractive indices of the quasi-D-shaped fiber.

\begin{tabular}{ccc|c|c}
\hline $\begin{array}{c}\text { Temperature } \\
\left({ }^{\circ} \mathrm{C}\right)\end{array}$ & $\begin{array}{c}\text { Wavelength } \\
(\mathrm{nm})\end{array}$ & $\mathrm{n}_{\mathrm{L}}$ & $\mathrm{n}_{\mathrm{s}}$ & $\mathrm{n}_{\mathrm{c}}$ \\
\hline 25 & $456.1 \mathrm{~nm}$ & 1.4605 & 1.4602 & 1.4612
\end{tabular}

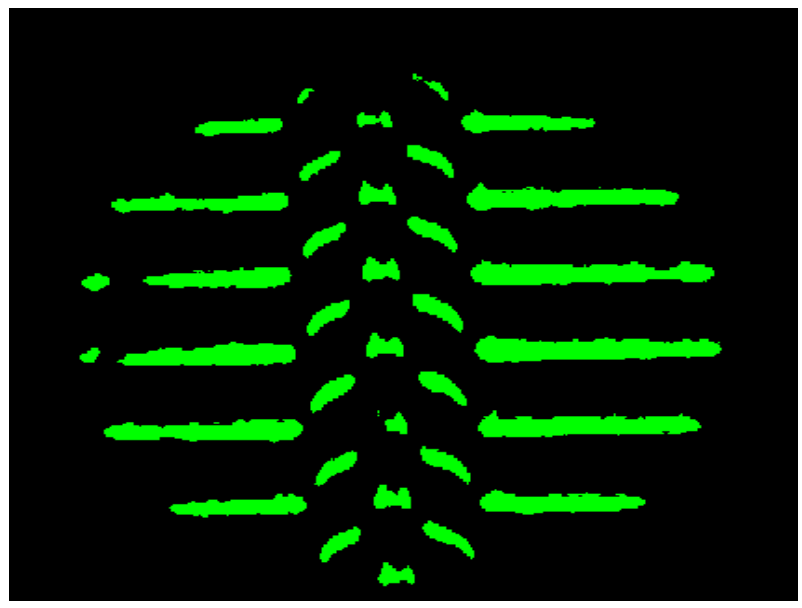

Fig. (4): Microinterferograms of multiple-beam Fizeau crossing quasi-D-shaped fiber in transmission.

\section{Conclusion:}

In conclusion we have derived a new mathematical formula for multiplebeam Fizeau fringes crossing quasi-D-shaped fiber. Numerical calculations for two different rotation angles have been presented. The experimental validation for the derived equations has been introduced. Good agreement between the theoretical and experimental results is given. This work is very useful for 
quality control for producing such fibers. The adjustment of the three basic transmission parameters refractive indices of core and cladding and core diameter are major of importance. The presented work can be applied for even smaller fiber dimensions.

\section{References}

1. R. EL-Agmy, W. Luethy, Th. Graf, H. P. Weber, Appl. Phys. B. 67, 23 (2003).

2. R. EL-Agmy, W. Luethy, Th. Graf, H. P. Weber, Electronics letters 39 (6), 507 (2003).

3. R. C. Faust, Proc. Phys. Soc., 67, 138 (2008).

4. S. M. Betrabet, K. P. R. Pillay and R. L. N. Lyendar, Text. Res. J. 33, 720 (1963).

5. A.A. Hamza, A.E. Belal, T.Z.N. Sokkar, H.M. EL-Dessouky, M.A. Agour, Optics and Lasers in Engineering, 45 (1), 145 (2007).

6. E.A. Galperin, Computers \& Mathematics with Applications, 56 (5), 1271 (2008).

7. M. J. Pluta, Microsc. 96, 309 (1971).

8. N. Barakat, A. A. Hamza, ,intrferometry of fibrous materials“, Adam Higler, Brisol, (1990).

9. Xiaojun Cao, Jianqiang Zhu, Yanhong Li, Qiang Lin, Optik - International Journal for Light and Electron Optics, 118 (10), 495 (2007).

10. M. M. El-Nicklawy, I. M. Fouda, J. Text. Inst. 71, 252 (1980).

11. Sanjib Chatterjee, Y. Pawan Kumar, Optics \& Laser Technology, 39 (3), 662 (2007).

12. M. M. El-Nicklawy, I. M. Fouda, J. Text. Inst. 71, 257 (1980).

13. Y.J. Rao, J. Jiang, C.X. Zhou, Sensors and Actuators A: Physical, 12 ( 2), 354 (2005).

14. M. M. El-Nicklawy, I. M. Fouda, K. A. El-Farahaty, Acta Phys. Pol. 67, 581 (1983).

15. M. M. El-Nicklawy, I. M. Fouda, A. M. Hassan, Poly. Test. 16, 33 (1996). 